\title{
COMPARATIVE AUDIT OF INDUCTION OF LABOUR AT FURNESS GENERAL HOSPITAL
}

\author{
R Ghani, SpR; MR Said, Staff Grade; \\ IY Hussein, Consultant; Department of Obstetrics \& Gynaecology; \\ BA Teague, Manager; E Stewart, Assistant, Clinical Audit Department, \\ Furness General Hospital
}

\section{INTRODUCTION}

Induction of labour (IOL) is the artificial initiation of uterine contractions prior to their spontaneous onset, leading to progressive dilatation, effacement of the cervix and delivery of the baby. The purpose of induction is to achieve benefit to the health of the mother and/or baby, greater than if the pregnancy continued. In recent years, the major fetal and maternal indications for IOL have not altered greatly. They still include prolonged pregnancy, pre-eclampsia, dysmaturity, antepartum haemorrhage, gross fetal abnormality, rhesus incompatibility, diabetes mellitus and fetal death in utero. In the UK the commonest indication is prolonged pregnancy. In addition, IOL is often performed now for cumulative indications, any of which if considered in isolation would probably constitute an insufficient reason: for example, increasing maternal age, previous infertility, poor obstetric history. Whatever the indication for induction, it is essential that gestational age is calculated accurately.

The rate of induction varies widely in different units, and between individual obstetricians within the same unit.

\section{AIM}

The aim of the audit was to determine the incidence, appropriateness and timeliness of IOL at Furness General Hospital during two distinct twelve-month periods (1994 and 1998/99) and to compare local practice to national guidelines.

\section{OBJECTIVES}

The objectives were to determine the main indications for intervention, the various methods of induction and outcome measures, such as mode of delivery, maternal blood loss, perineal trauma and fetal outcome.

\section{METHODOLGY}

The labour ward birth register was used as the data source for the 1994 audit to identify women who underwent IOL during the period 1 January to 31 December 1994. The maternity casenotes were extracted and a proforma was designed for retrospective data collection. The proforma was then revised for prospective data collection on the maternity and labour wards during the period 1 August 1998 to 31 July 1999. A number of medical casenotes were reviewed for data validation purposes. The information was analysed by the clinical audit department using Epi Info V6.04b (World Health Organisation database and statistical package).

\section{RESULTS}

\section{Incidence}

During 1994 there were 305 interventions at Furness General Hospital and a total of 1266 deliveries in the maternity unit, representing an incidence of 241 per 1000 . During a twelvemonth period in 1998/99 there were 252 interventions and a total of 1125 deliveries in the maternity unit, representing an incidence of 224 per 1000 . When compared to the 1994 data the 1998/99 statistics reflect a slight decline in the local incidence. There was a fairly even distribution in the number of procedures performed by each consultant team.

\section{Characterisation}

The age distribution of the patients was 14-45 years (median 26 years) in 1994 and 16-46 years (median 27 years) in 1998/99 (Figure 1).

The incidence of multiparous women was $53 \%$ and primiparous $47 \%$, which was similar to the 1994 audit $(57 \%$ and $43 \%$ respectively).

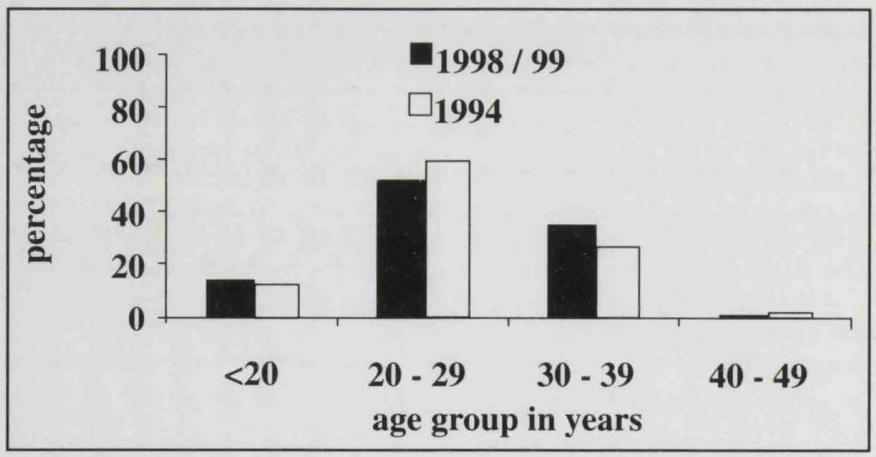

Figure l Age distribution

The gestational age at IOL was over a wide range with $46 \%$ of patients $>40$ weeks in 1994 and $41 \%$ in $1998 / 99$. It was also noted that there was a $4 \%$ increase at 38 weeks' gestation in 1998/99 (Figure 2).

\section{Main indications for intervention}

There was an overlap in the main indications for intervention. In $198 / 99$ these were postdate $(46 \%)$, pregnancy-induced hypertension (20\%) and social/maternal request (20\%). Other reasons included poor obstetric history, co-existing medical or surgical problems, advanced maternal age (19\%), intra- 


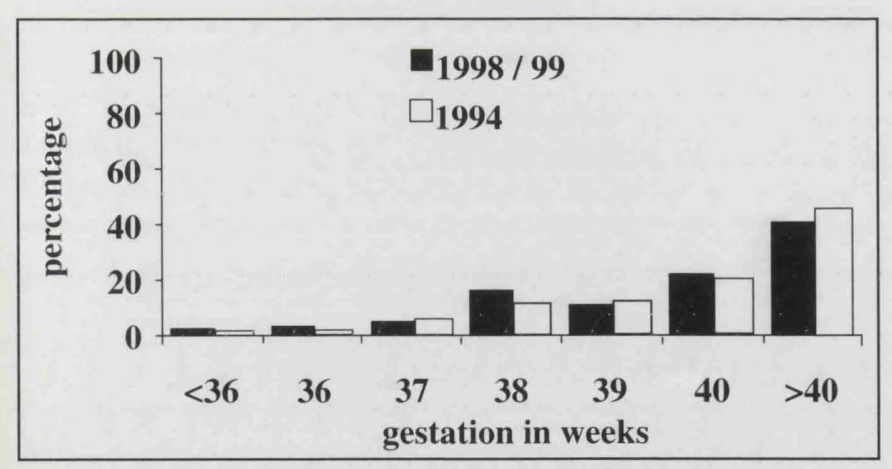

Figure 2 Gestational age at $I O L$

uterine growth retardation ( $8 \%)$ and reduced fetal movements $(5 \%)$. Figure 3 compares the overall indications for intervention in 1994 and 1998/99.

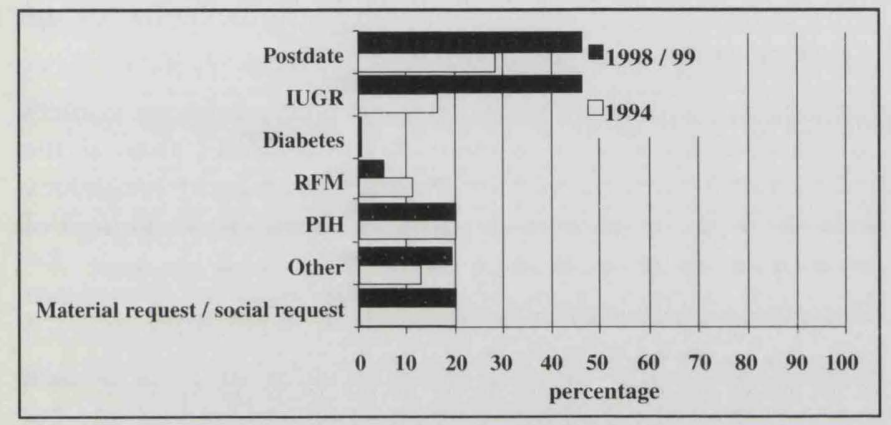

Figure 3 Main indications for intervention

When the reason for IOL was postdate, $53 \%$ took place $8-14$ days after the expected date of delivery (47\% in 1994).

When social/maternal request was the reason for $\mathrm{IOL}$, in $1998 / 9937 \%$ of these took place at 38 weeks' gestation, whilst beyond 40 weeks the figure fell to $13 \%$ (Figure 4).

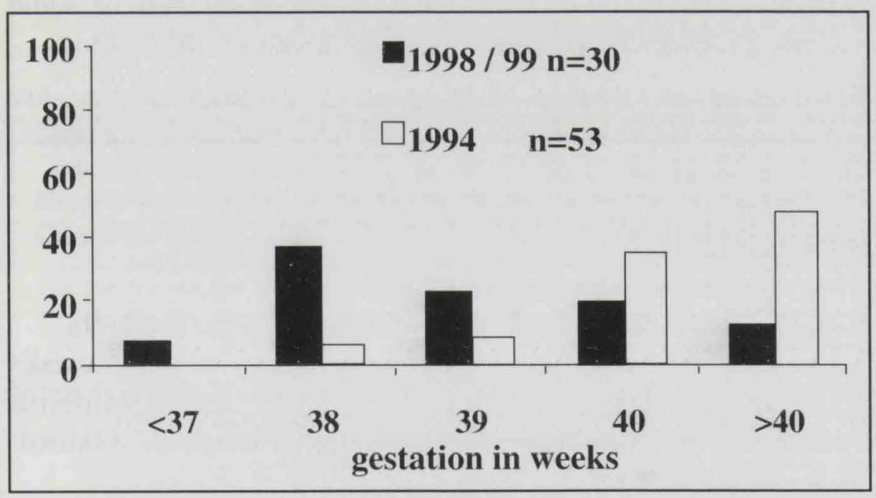

Figure 4 Social/maternal request

\section{Timing and method of induction}

In 1998/99 there were $71 \%$ of primipara induced at or beyond 40 weeks' gestation, whilst $19 \%$ were between 38 and 40 weeks' gestation. Fifty-six percent of multipara induced were at or beyond 40 weeks' gestation, whilst $34 \%$ were between 38 and 40 weeks' gestation.

In $1998 / 99,51 \%$ of primipara and $60 \%$ of multipara were induced by Prostaglandin E2 alone, compared to $29 \%$ and $36 \%$ in 1994. The estimated cost of Prostin medication was $£ 7479$.

Figure 5 illustrates that there was less combination analgesia administered to the women in 1998/99 with a corresponding increase in Pethidine and Entonox.

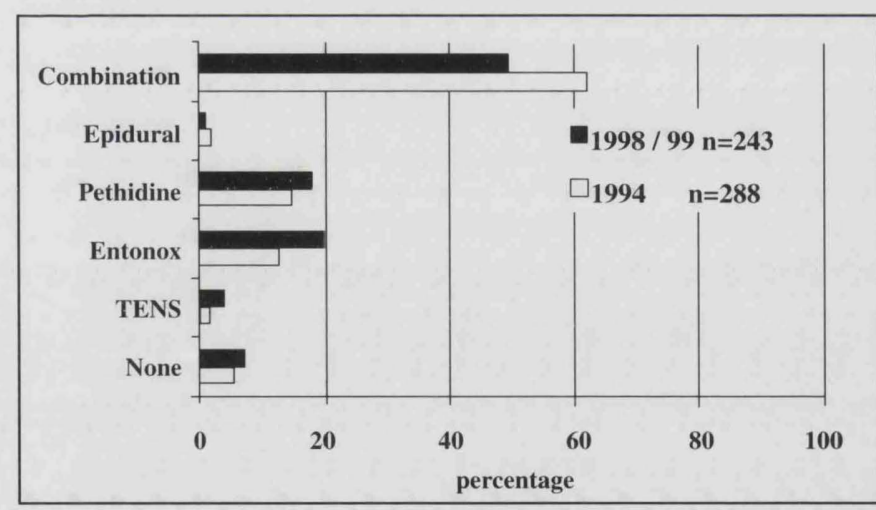

Figure 5 Analgesia

\section{Progress of labour}

In 1998/99 the range in the onset of labour was between one and $96+$ hours (mean 26 hours). Eighty percent started labour within 24 hours of induction, $78 \%$ had a first stage $<6$ hours, $80 \%$ had a second stage $<60$ minutes and $97 \%$ had a third stage $<30$ minutes.

\section{Outcome - maternal}

Spontaneous vaginal delivery occurred in $81 \%$ of patients in $1998 / 99$ and $77 \%$ in 1994 . Thirteen percent of inductions resulted in caesarean section (Figure 6).

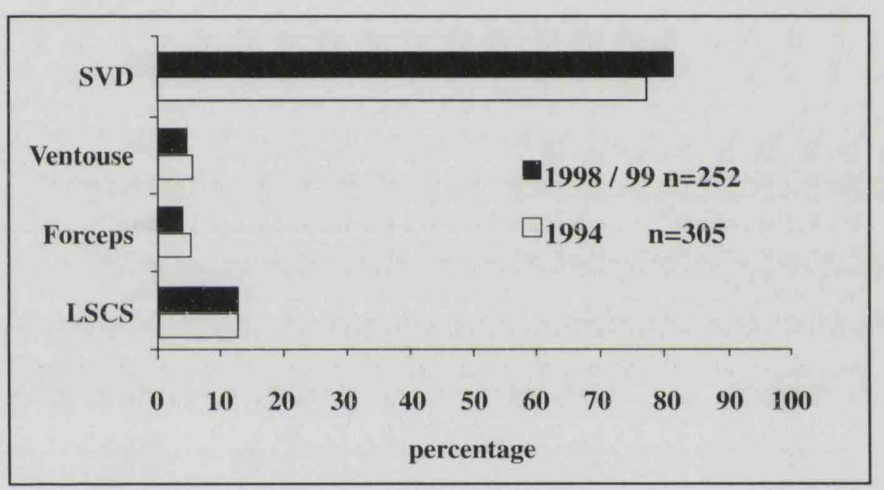

Figure 6 Mode of delivery

The main reasons for operative intervention in 48 patients $(19 \%)$ were failure to progress (primipara $61 \%$ and multipara $50 \%$ ) and fetal distress (primipara 36\% and multipara 25\%). The figures were similar for both audits.

The blood loss was estimated to be $<500 \mathrm{ml}$ in $80 \%$ of women, between $500-1000 \mathrm{ml}$ in $16 \%$ and $>1000 \mathrm{ml}$ in $4 \%$. The overall primary postpartum haemorrhage rate was $20 \%$ in 1998/99 compared to only $13 \%$ in 1994 .

Episiotomy was performed in 19\% of women in 1998/99, which was much lower than the 1994 figure of $25 \%$.

There has been a decline in the incidence of second degree $(16 \%)$ and third degree $(1 \%)$ lacerations, but an increase in first degree (28\%) lacerations since 1994.

\section{Outcome - fetal}

There were two women induced for intra-uterine fetal death in 1994 but no stillbirths in either audit. Figures 7a and 7b compare the distribution of the Apgar scores at one minute and five minutes.

The admission rate to SCBU remained constant at $10 \%$ in 1994 and 1998/99. 


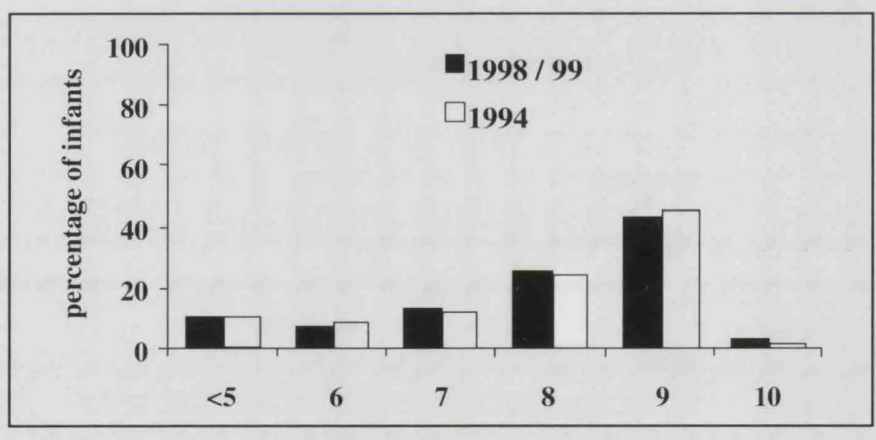

Figure 7a Apgar at 1 minute

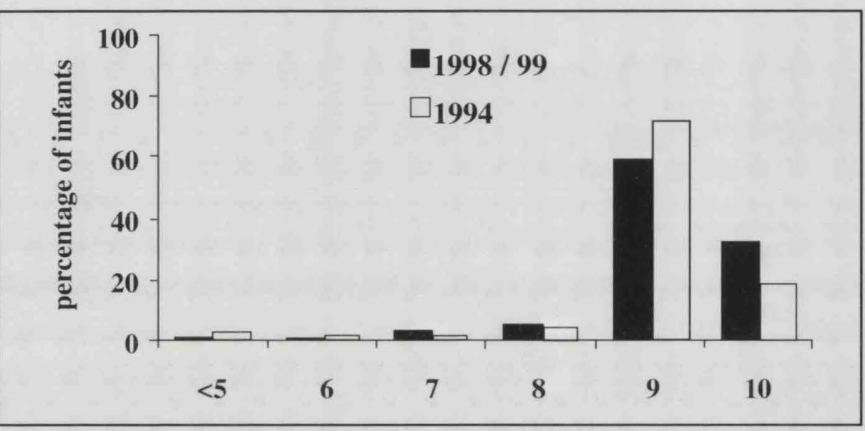

Figure $7 b$ Apgar at 5 minutes

\section{DISCUSSION}

The local rate for IOL for $1998 / 99$ was $22 \%$, which is consistent with national statistics, which show a wide variation of rates between $10 \%$ and $25 \%$. There was a marginal fall from the 1994 figure of $24 \%$. The procedures were performed on a comparable number of primiparous and multiparous women. The commonest indication for IOL was prolonged pregnancy. There is evidence that IOL beyond 41 weeks is associated with lower rates of caesarean section, operative delivery, meconium stained liquor, macrosomia and fetal/neonatal death ${ }^{(2)}$. Women are tending to have their families at a later age ${ }^{(3)}$ and the effect of this is portrayed in Figure 1.

In 1998/99 there was a significant increase in women induced at 38-39 weeks' gestation for maternal request. As a result the number of normal vaginal deliveries fell in this group, whilst the caesarean section rate rose from $8 \%$ in 1994 to $13 \%$ in $1998 / 99$. The cervix tends to be less favourable at 38-39 weeks' gestation than at 41 weeks $^{(4)}$ and consequently it is more difficult to initiate labour. The process can take several days and in some instances may not work at all. Mothers-to-be have expectations of a speedy induction process and often get demoralised with prolonged induction. Many will request delivery by caesarean section after a few days of induction.

The majority of women were delivered normally $(81 \%$ in 1998 and $77 \%$ in 1994), whilst the caesarean section rate amongst the induction group remained constant at $13 \%$ for both periods. There was a decline in the number of forceps deliveries, in favour of ventouse. This is consistent with the national trend ${ }^{(5)}$

There was a significant rise in the incidence of primary post-partum haemorrhage, principally from uterine atony.

There was a welcome decline in episiotomies performed and second and third degree lacerations. Fetal outcome was unaltered in the two audits and remained favourable.

\section{CONCLUSION}

The comparative audit confirmed that IOL continues to be a relatively safe procedure with favourable feto-maternal outcome. However it did show that social IOL at 38-39 weeks' gestation was associated with a significant increase in the caesarean section rate

\section{RECOMMENDATIONS}

The following recommendations were agreed and formulated to ensure continuing improvement in feto-maternal outcome:-

- Women should always be informed of the benefits and risks associated with IOL versus that of expectant management and their preferences regarding these policies should be respected.

- Careful documentation relating to the indications for the induction must be maintained.

- Induction should be recommended when delivery confers clear benefit to the woman or baby greater than if the pregnancy continues. This includes prolonged pregnancy ( $>41$ weeks' gestation), although accurate dating of gestational age is essential.

- The decision for IOL must be made at senior level.

- Social inductions should only take place after the woman has been fully informed of the risks of induction, but patients' requests should be respected.

- When IOL is undertaken in a case of previous caesarean section, it is important that the decision and subsequent events are under the close supervision of staff at consultant level.

- Fetal surveillance following induction of labour is mandatory.

- Vigilance for primary post-partum haemorrhage is required at all times. Active management is advised and in some cases prophylactic measures with syntocinon infusion.

- Re-audit to ensure that these recommendations are followed, with resultant improved feto-maternal outcome.

\section{REFERENCES}

1 RCOG. Induction of Labour, Guidline No 16, July 1998

2 Crowley P. Electrive induction of labour at $41+$ weeks gestation. In: The Cochrane Pregnancy and Childbirth Database. The Cochrane Collaboration; Issue 2, Oxford: Update Software 1995

3 ONS England \& Wales. Trends in epidemiology \& women's health. CEMD 1994-1996

4 Bishop EH. Pelvic scoring for elective induction. Obstet Gynecol 1964;24:266-8

5 Meniru GI. An analysis of recent trends in vacuum extraction and forceps delivery in the United Kingdom. Br J Obstet Gynaecol 1996;103:168-70

\section{Acknowledgement}

The authors are indebted to Stella McDowell, Deputy Midwifery Ward Manager, Maternity Unit, Furness General Hospital, who performed the endless task of data collection and validation for the 1994 audit. The authors are similarly indebted to the midwifery and medical staff who recorded and validated the data for the 1998/99 audit. 